\title{
Thyroid Gland Black Follicular Adenoma
}

National Cancer Institute

\section{Source}

National Cancer Institute. Thyroid Gland Black Follicular Adenoma. NCI Thesaurus. Code C155958.

A thyroid gland follicular adenoma seen in patients treated with minocycline. The tumors have black discoloration visible on macroscopic examination and cytoplasmic accumulation of black pigment. (WHO 2017) 